\title{
Protective effect of lentivirus-mediated siRNA targeting ADAMTS-5 on cartilage degradation in a rat model of osteoarthritis
}

\author{
XIANGYU CHU, HONGBO YOU, XUEFENG YUAN, WENBIN ZHAO, WENKAI LI and XIN GUO \\ Department of Orthopedics, Tongji Hospital, Tongji Medical College, \\ Huazhong University of Science and Technology, Wuhan, Hubei 430030, P.R. China
}

Received January 25, 2013; Accepted March 12, 2013

DOI: $10.3892 /$ ijmm.2013.1318

\begin{abstract}
The etiology of osteoarthritis (OA) is complex and multifaceted. Osteoarthritis is a chronic and progressive disease of the joints that is characterized by the degradation of articular cartilage. A disintegrin and metalloproteinase with thrombospondin motifs-5 (ADAMTS-5) is the major aggrecanase in cartilage. The aim of this study was to evaluate the effect of ADAMTS-5 knockdown on cartilage degradation. Rat articular chondrocytes were transfected with lentivirus-mediated ADAMTS-5 small interfering RNA (siRNA) or with empty vector control plasmid DNA (as the control). The suppression efficiency was measured using real-time polymerase chain reaction (RT-PCR) and western blot analysis. We then selected the most effective siRNA (siRNA1) and constructed the lentivirus-mediated siRNA targeting ADAMTS-5 for stable transfection. An animal model of OA was created using male Sprague-Dawley rats. OA was induced by performing anterior cruciate ligament transection (ACL-T) and partial medial meniscectomy (PM). The animals $(n=80$, weight 250-300 g) received an intra-articular injection of the empty vector control plasmid DNA or lentivirus-mediated ADAMTS-5 siRNA1 (20 $\left.\mu \mathrm{l}, 1 \times 10^{8} \mathrm{TU} / \mathrm{ml}\right)$. The progression of OA was analyzed using Osteoarthritis Research Society International (OARSI) scores. Compared with the control, ADAMTS-5 gene expression was decreased by approximately $80 \%$ by siRNA 1 in a monolayer culture of chondrocytes. The intra-articular injection of lentivirus-mediated ADAMTS-5 siRNA1 in vivo resulted in the downregulation of ADAMTS-5 protein expression and improved OARSI scores $(\mathrm{p}<0.05)$. A single injection of lentivirus-mediated ADAMTS-5 siRNA prevented the degra-
\end{abstract}

Correspondence to: Dr Hongbo You, Department of Orthopedics, Tongji Hospital, Tongji Medical College, Huazhong University of Science and Technology, 13 Hangkong Road, Wuhan, Hubei 430030, P.R. China

E-mail: youhongbo003@yahoo.com.cn

Key words: osteoarthritis, a disintegrin and metalloproteinase with thrombopondin motifs-5, RNA interference, cartilage dation of articular cartilage. This method may provide a novel therapeutic strategy for the treatment of human OA.

\section{Introduction}

Osteoarthritis (OA), also known as degenerative arthritis, is a common chronic and progressive disorder of the joints in aging populations (1). It is a leading cause of pain and disability. OA is characterized by the degeneration and loss of articular cartilage (2). Although the initiation of OA may be multifactorial, the articular cartilage degradation seems to be a direct result of infinite proteolytic extracellular matrix destruction. The cartilage extracellular matrix has a rich distribution of aggrecan and type 2 collagen. The loss of aggrecan is considered a reversible event in OA cartilage degradation (2). A previous study suggested that a disintegrin and metalloproteinase with thrombospondin motifs-5 (ADAMTS-5) is the major aggrecanase in cartilage (3), and that the inhibition of the expression of the ADAMTS-5 gene is likely to represent a novel therapeutic strategy for the treatment of OA by preventing cartilage aggrecan breakdown. A previous study on a murine model of OA revealed a significantly reduced level of cartilage degradation in ADAMTS-5 knockout mice compared with wild-type mice (4). However, this study proved to be costly and time consuming. Since RNA interference (RNAi) was initially discovered by Fire et al (5), this sequence-specific gene-silencing technology has received a great deal of attention from the scientific community $(6,7)$. This method provides a specific, effective and simple way of silencing a target gene (8). Small interfering RNA (siRNA) can be delivered by stable viral-mediated transfection. Lentiviral vectors derived from HIV-1 are able to infect a broad variety of cells, including dividing and non-dividing cells (9). siRNA can stably integrate into the host genome, resulting in the long-term expression of the transgene (10).

In the present study, we investigated the protective effect of lentivirus-mediated siRNA targeting ADAMTS-5 on cartilage degradation in a rat model of OA.

\section{Materials and methods}

Animals. In this study, male Sprague-Dawley rats (acquired from Wuhan University Animal Experiment Center, Wuhan, 
China) were allowed to reach a body weight of 250-300 g before surgery and were kept under controlled environmental conditions (i.e., $22^{\circ} \mathrm{C}$, 40-60\% relative humidity). Food and water were provided ad libitum. All in vivo experiments were performed in accordance with the Hubei Province Regulations for the Administration of Experimental Animals. The animal protocols were approved by the Animal Care and Use Committee of Tongji Medical College, Wuhan, China.

Isolation and cultivation of rat articular chondrocytes. Articular cartilage from the knees of male Sprague-Dawley rats (15-20 g, 1 week old) was dissected after the animals were sacrificed by cervical dislocation. Tissues were cut into $1-\mathrm{mm}^{3}$ samples, and the cells were isolated using a sequential proteinase and collagenase digestion technique, as previously described by $\mathrm{Hu}$ et al (11). Cartilage samples were collected and washed twice for $10 \mathrm{~min}$ in Dulbecco's modified Eagle's medium (DMEM) supplemented with $44 \mathrm{mM} \mathrm{NaHCO}$ (Sigma Aldrich, St. Louis, MO, USA), $20 \mathrm{mM} \mathrm{N}$-2-hydroxyethylpiperazine$\mathrm{N}$-2-ethanesulfonic acid (HEPES), $\mathrm{pH} 7.4$, containing the following antibiotics: $100 / \mathrm{ml}$ penicillin $\mathrm{G}$ sodium, $\mathrm{MgCl}_{2}$ (0.23 mmol/1, Sigma Aldrich) and $100 \mu \mathrm{g} / \mathrm{ml}$ streptomycin sulfate (medium A) (12).

The cartilage sections were initially treated with trypsin (0.25 g/ml, Sigma Aldrich) for $30 \mathrm{~min}$ at $37^{\circ} \mathrm{C}$. Trypsin was inhibited by incubation in medium A containing $12 \%$ fetal calf serum (FCS; Gibco, Invitrogen, Grand Island, NY, USA). Digested tissue was transferred into medium A plus $12 \%$ FCS containing $0.2 \%$ collagenase 2 and $0.5 \%$ hyaluronidase, and incubated for $4 \mathrm{~h}$ at $37^{\circ} \mathrm{C}$ with constant stirring. A $100-\mu \mathrm{m}$ nylon-mesh strainer was used for filtering the digestion mixture. Cells were collected by centrifugation $(1,500 \mathrm{rpm}, 5 \mathrm{~min})$ and washed twice with medium $\mathrm{A}$. The chondrocytes were suspended in medium $\mathrm{A}$ and expended in monolayer culture in medium A plus $12 \% \mathrm{FCS}$ at $37^{\circ} \mathrm{C}$ under a humidified atmosphere containing $5 \% \mathrm{CO}_{2}$. The cells were seeded in a 6-well plate at a density of $4 \times 10^{5} /$ well prior to transfection.

Establishment of siRNAs for ADAMTS-5 oligonucleotide and transient transfection. Three pairs of RNA oligonucleotides specific for the ADAMTS-5 coding region were designed using the online tool (http://www.invitrogen.com/site/us/en/home/ References/Ambion-Tech-Support/rnai-sirna/general-articles/sirna-design-guidelines.html) and were constructed from a completely homologous region of sequences in the human, mouse and rabbit ADAMTS-5 gene from the National Center for Biotechnology Information (NCBI) website (http://www. ncbi.nlm.nih.gov/index.html). The sequences for the ADAMTS-5 siRNA oligonucleotides used in all further experiments were as follows: sense, 5'-UCGAUCCCUAGCUGUCUUUTT-3' and antisense, 5'-TTAGCUAGGGAUCGACAGAAA-3' for siRNA1; sense, 5'-CACGCAUCCUGCAUGUCUATT-3' and antisense, 5'-TTGUGCGUAGGACGUACAGAU-3' for siRNA2; and sense, 5'-CAGGAUGGAAACAGGAAAUTT-3' and antisense, 5'-TTGUCCUACCUUUGUCCUUUA-3' for siRNA3. These siRNAs were chemically synthesized by GenePharma Co. Ltd. (Shanghai, China). After $6 \mathrm{~h}$ of incubation, we detected the transfection efficiency by fluorescence analysis. The cells were harvested after $48 \mathrm{~h}$ of incubation, and the protein and mRNA expression level of ADAMTS-5 was assessed by western blot analysis and quantitative real-time PCR (qRT-PCR), respectively as previously described (13).

$R N A$ isolation and $q R T-P C R$. As previously described by Hummon et al (14), total cellular RNA was extracted and isolated from the chondrocytes after $48 \mathrm{~h}$ of transfection using TRIzol reagent (Invitrogen) according to the manufacturer's instructions. The RNA concentration was measured using the BioPhotometer Plus (Eppendorf, Hamburg, Germany), and all samples were stored at $-80^{\circ} \mathrm{C}$ until further treatment.

Complementary DNA (cDNA) was obtained by reverse transcription of $1 \mu \mathrm{g}$ total RNA using specific target primers. These primers were 18-20 mers, designed using Primer 5 software (Premier Biosoft, Palo Alto, CA, USA) to amplify the rat ADAMTS-5 gene and the housekeeping gene, glyceraldehyde-3-phosphate dehydrogenase (GADPH). The sequences for the primers used in this study were as follows: forward, 5'-TGTGGTGCGCCAAGGCCAAA-3' and reverse, 5'-CCCT GTGCAGTAGCGGCCAC-3' for ADAMTS-5; and forward, 5'-CTCATGACCACAGTCCATGC-3' and reverse, 5'-TTCAGC TCTGGGATGACCTT-3' for GADPH. cDNA was synthesized in a total volume of $20 \mu \mathrm{l}$ at $42^{\circ} \mathrm{C}$ for $1 \mathrm{~h}$ using the Toyobo high-efficiency reverse transcriptase kit (Toyobo Co. Ltd., Osaka, Japan). The mRNA expression levels were determined by qRT-PCR and FastStart DNA SYBR-Green reagent (Roche, Basel, Switzerland). qRT-PCR was performed as follows: $5 \mathrm{~min}$ at $95^{\circ} \mathrm{C}(1 \mathrm{cycle}), 20 \mathrm{sec}$ at $94^{\circ} \mathrm{C}, 20 \mathrm{sec}$ at $59^{\circ} \mathrm{C}, 20 \mathrm{sec}$ at $72^{\circ} \mathrm{C}$, and reading plate (40 cycles). Raw data of cycle threshold $(\mathrm{Ct})$ values for ADAMTS-5 in each group were normalized to those of GADPH. ADAMTS-5 mRNA levels were determined by column diagram analysis.

Western blot analysis. The protein expression of ADAMTS-5 was evaluated by western blot analysis. Total proteins from chondrocytes in each group were extracted using a protein extraction kit (ProMab Biotechnologies, Albany, CA, USA) $48 \mathrm{~h}$ after transfection. The cellular proteins were then denatured and resolved by $12 \%$ sodium dodecyl sulfate polyacrylamide gel electrophoresis (SDS-PAGE). After electrophoretic separation, the proteins were transferred onto nitrocellulose membranes (Invitrogen). The membranes were blocked with 5\% non-fat skim milk in Tris-buffered saline (TBS) containing Tween-20 buffer at normal temperature for $1 \mathrm{~h}$. The membranes were then incubated overnight with anti-ADAMTS-5 (ab41037; Abcam, Cambridge, UK) or anti-GADPH (sc-365062; Santa Cruz Biotechnology Inc., Santa Cruz, CA, USA) monocolonal antibody at $4^{\circ} \mathrm{C}$. After being washed, the protein of interest was visualized using the BeyoECL Plus enhanced chemiluminescence western blotting detection system (Beyotime, Shangshai, China). The expression level of ADAMTS-5 was calculated using Gel-Pro Analyzer 4.0 Image Analysis Software (Media Cybernetics, Inc., Bethesda, MD, USA) and was normalized to the GADPH level.

Construction of lentiviral vector expressing siRNA targeting ADAMTS-5. siRNA1 was converted into short hairpin RNA (shRNA) according to the stem-loop-stem structure followed by the addition of AgeI [R0552v, New England Biolabs (NEB), Ipswich, MA] and EcoRI (NEB, R0101v) restriction sites at the $5^{\prime}$ and $3^{\prime}$ end, respectively. DNA oligonucleotides 
(GeneChem Co. Ltd., Shanghai, China) were annealed and inserted into pGCSIL-GFP (GeneChem) vectors by double digestion with $A g e I$ and EcoRI with T4 DNA ligase (NEB, M0202v). The vectors were transformed into DH5 $\alpha$-competent Escherichia coli cells; we then confirmed the insertions by restriction enzyme analysis and DNA sequencing.

Lentiviruses were co-transfected into 293T cells using Lipofectamine 2000 (Invitrogen) and were used to infect the chondrocytes at a multiplicity of infection (MOI) of 40 (15).

Surgically induced OA in a rat model. Briefly, the animals were anesthetized using chloral hydrate $(350 \mathrm{mg} / \mathrm{kg})$. OA pathogenesis was surgically induced in 80 of the rats, using open surgery involving anterior cruciate ligament transection (ACL-T) and partial medial meniscectomy (PM), implemented as previously described by Appleton et al (16). Surgery was performed only on the right knee. All animals were administered antibiotics and analgesics following surgery. The animals were randomly divided into 4 groups: a negative control group, an experimental group, a blank control group and a sham operated group. The experimental group was administered an intra-articular injection of lentivirus-mediated ADAMTS-5 siRNA $\left(20 \mu 1,1 \times 10^{8}\right.$ $\mathrm{TU} / \mathrm{ml}$ ). However, the negative control group was injected with the same dose of empty vector control plasmid DNA. The sham operated group underwent a similar incision in the right knee joint, although ACL-T and PM were not performed; the blank control group did not undergo a surgical procedure.

Four animals were used per time point in each treatment group. Forced mobilization (FM) was used to accelerate OA onset and severity, beginning 1 week prior to surgery. A rotating animal cage apparatus $(17,18)$ with divided lanes was manufactured for FM (Fig. 1A). This device rotated toward the rats at a rate of $5 \mathrm{rpm}$ and forced the animals to flex and extend the knee joint as they walked in the cage (Fig. 1B). Each rat completed a 30-min session of FM each week on Tuesdays, Thursdays and Saturdays. The animals were sacrificed at 2, 4, 8, 12 and 16 weeks after surgery. The tibiofemoral joints were dissected from each rat and were processed for histological evaluation.

Western blot analysis in vivo. The protein expression of ADAMTS-5 in vivo was evaluated by western blot analysis. Total ADAMTS-5 protein in each experimental group was extracted using a protein extraction kit (ProMab) at 2, 4, 8, 12 , and 16 weeks after transfection. The cellular proteins were then denatured and resolved by $12 \%$ SDS-PAGE. Following electrophoretic separation, proteins were transferred onto nitrocellulose membranes (Invitrogen). The membranes were blocked with 5\% non-fat skim milk in TBS containing Tween-20 buffer at normal temperature for $1 \mathrm{~h}$. The membranes were then incubated overnight with anti-ADAMTS-5 (ab41037; Abcam) or anti-GADPH (sc-365062; Santa Cruz Biotechnology Inc.) monocolonal antibody at $4^{\circ} \mathrm{C}$. After being washed, the protein of interest was visualized using the BeyoECL Plus enhanced chemiluminescence western blotting detection system. The expression level of ADAMTS-5 was calculated using Gel-Pro Analyzer 4.0 Image Analysis Software and normalized to the GADPH level.

Processing and scoring of histological samples. The knee cartilage samples were fixed in $4 \%$ paraformaldehyde in phos- phate-buffered saline for $24 \mathrm{~h}$ at each time point; the cartilage samples were then decalcified in $10 \%$ nitric acid in neutralbuffered formalin (containing 4\% formaldehyde) for 24-72 $\mathrm{h}$. Finally, the decalcified samples were embedded in wax blocks for sectioning. Serial sections $(6 \mu \mathrm{m})$, through the mid-joint edge, were stained with toluidine blue. A total of 5 stained sections per sample were used for histological scoring.

The progression of OA in all samples was assessed and compared according to the new Osteoarthritis Research Society International (OARSI) Cartilage Histopathology Assessment System (OOCHAS) (19). The system uses a 24-point scale based on a combination of OA grade (0-6 points) and OA stage (0-4 points). Both the tibia and femur tissues were evaluated independently in 5 stained sections from each sample by an observer blinded to the histological data.

Statistical analysis. Statistical analysis software [Statistical Package for the Social Sciences (SPSS) version 19.0, IBM, Armonk, NY, USA] was used for all statistical tests. OOCHAS histological grading and staging scores were performed with analysis of variance (ANOVA) to determine whether the time point was significant. In addition, the Student-Newman-Keuls test was used to compare differences between groups. A p-value $<0.05$ was considered to indicate a statistically significant difference.

\section{Results}

Morphological observation and identification of rat articular chondrocytes. Original generation chondrocytes are small and have a uniform distribution. These cells are almost triangular or polygonal and have strong refractive indices. The cytoplasm shows a rich distribution of secretory vesicles, endoplasmic reticulum and Golgi apparatus (Fig. 2A). Immunohistochemistry revealed higher levels of type 2 collagen in the nuclear membrane edge (Fig. 2B).

Effect of siRNA on rat ADAMTS-5 gene expression in rat chondrocytes. Three different siRNA constructs, siRNA1, siRNA2 and siRNA3, were used to silence the mRNA expression of ADAMTS-5 in the rat chondrocytes. A non-targeting, scrambled siRNA was used as the control. The transfection efficiency was observed under a fluorescence microscope $6 \mathrm{~h}$ following transfection. In the siRNA1 group, the green fluorescence cells were considered to be transfected successfully (Fig. 3A and B). At $48 \mathrm{~h}$ after transfection, all animals in the 3 experimental groups that had received ADAMTS-5 siRNA showed a downregulation in the constitutive mRNA expression of ADAMTS-5 (Fig. 3C). The ADAMTS-5 mRNA expression in the siRNA1 group $(1.10 \pm 0.16)$ was significantly lower than that in the negative control group $(5.54 \pm 0.68)$ and the blank control group $(5.54 \pm 0.62)(\mathrm{p}<0.05)$.

Western blot analysis showed that the protein expression level of ADAMTS-5 in the siRNA1 group [integrated optical density (IOD) value, 133.73] was significantly lower than that in the negative control group (IOD value, 323.95) and the blank control group (IOD value, 320.79) (Fig. 3D). We selected the most effective siRNA (siRNA1) and constructed the lentivirus-mediated siRNA targeting ADAMTS-5 for stable transfection. 
A

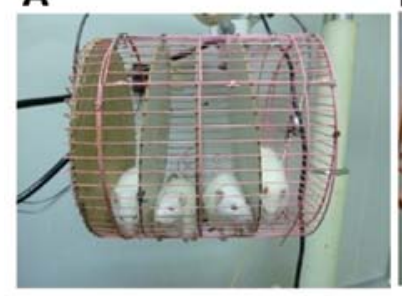

D

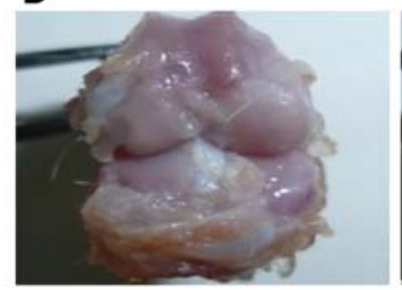

B

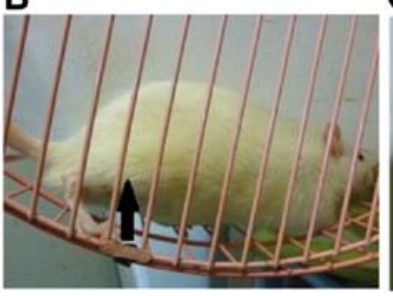

E

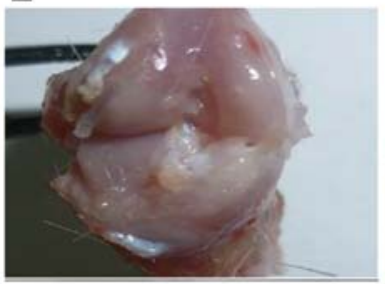

C

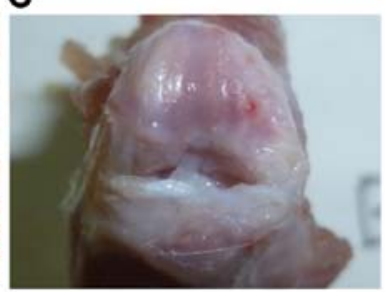

$\mathbf{F}$

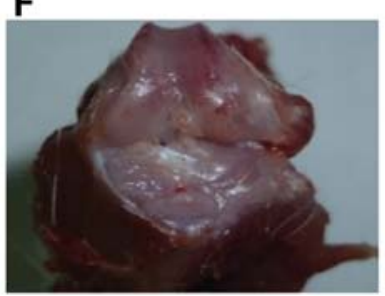

Figure 1. Forced mobilization (FM) apparatus and macroscopic analysis of joint degradation. (A) Animals walked in a rotating cage for 30 min, 3 times/week. (B) FM forced the maximal extension of the joint (black arrow). Knee joints were dissected at 2 and 4 weeks following surgery. Representative images from (C) the control group and (E) the blank group at 2 weeks, and (D) the control group and (F) the blank group at 4 weeks are shown. A smooth and glassy cartilage was observed in the blank group, while in the control group, surface abrasions and fibrotic tissue were observed.

A

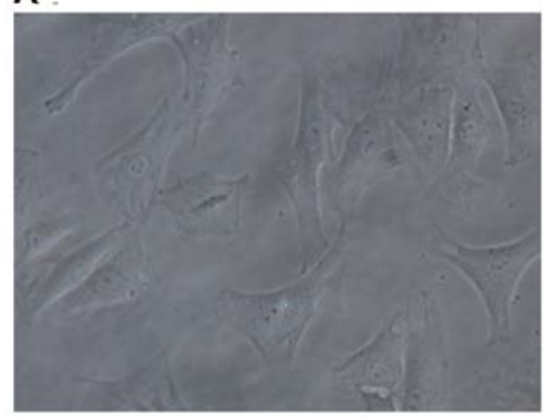

B

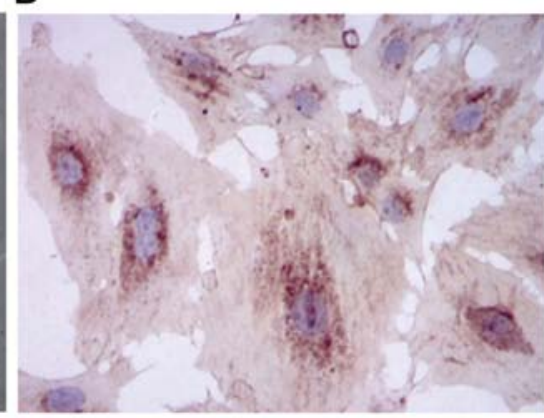

Figure 2. Microscopic observations of normal rat cartilage chondrocytes and the expression of type 2 collagen. (A) Chondrocytes (magnification, $\mathrm{x} 200$ ), closely spaced in monolayer culture, are almost polygonal and have short spindles. (B) Immunohistochemistry (magnification, $\mathrm{x} 400$ ) showed that higher levels of type 2 collagen were distributed in the nuclear membrane edges (brown particles).

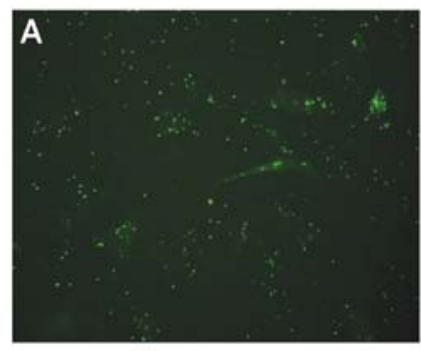

C

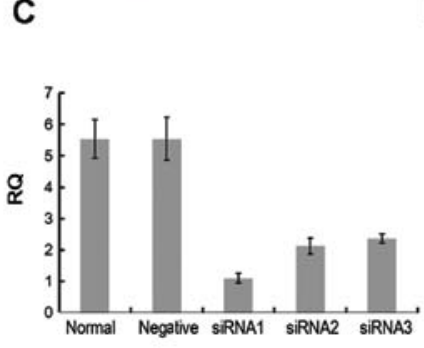

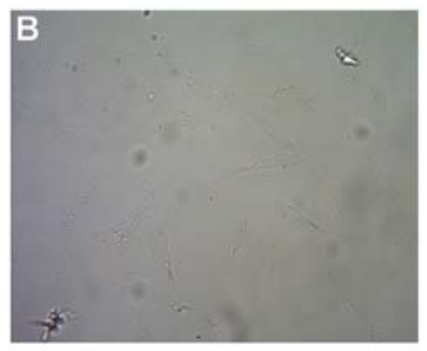

D

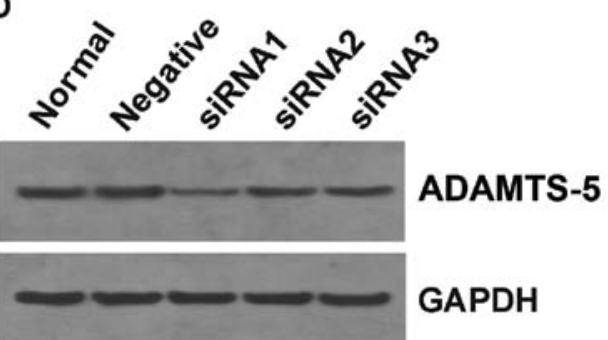

Figure 3. Transfection was successful; mRNA and protein levels of ADAMTS-5 were downregulated. (A) Green fluorescence image of cells transfected with ADAMTS-5 siRNA1 under a fluorescence microscope (magnification, x100). (B) The same chondrocytes under an electron microscope. (C) Column diagram analysis for ADAMTS-5 mRNA expression. ADAMTS-5 siRNA1 resulted in a significant reduction in the mRNA level of ADAMTS-5 in chondrocytes. RQ, relative quantity. (D) Western blot analysis for ADAMTS-5 protein expression. ADAMTS-5 protein expression was significantly downregulated in the siRNA1 group. 


\section{A}

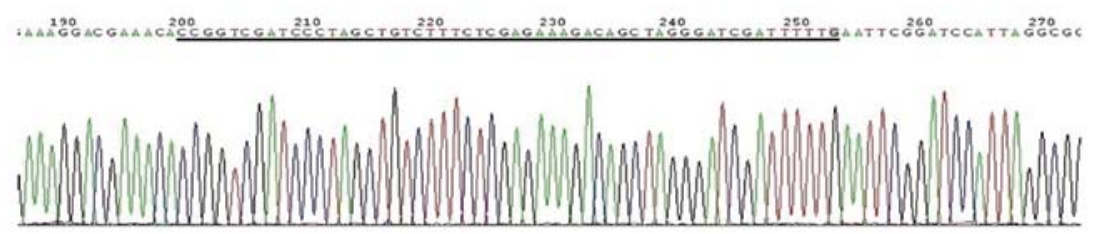

B
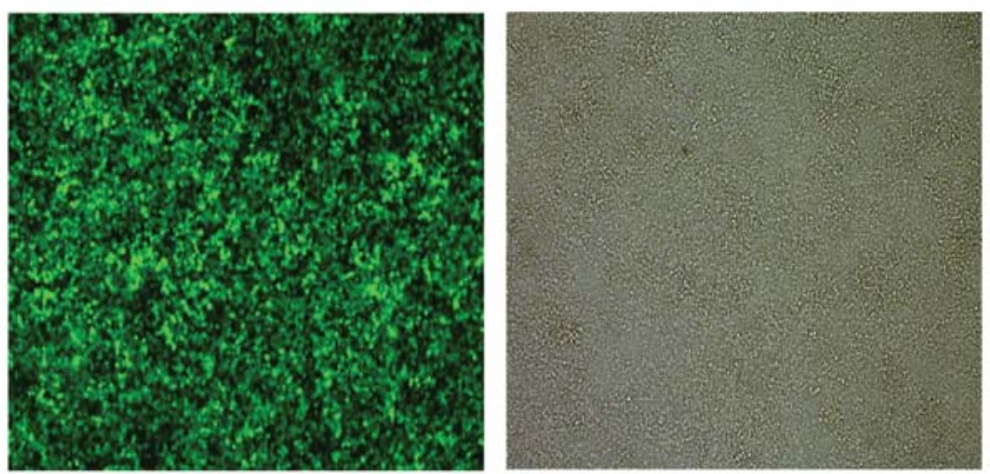

Figure 4. Confirmation of correct insertion of shRNA cassettes by DNA sequencing and chondrocyte infection. (A) DNA sequencing demonstrates the successful insertion of shRNA cassettes. (B) Green fluorescence imaging of cells infected with recombinant vectors under a fluorescence and electron microscope (left and right panel, respectively; magnification, x100).

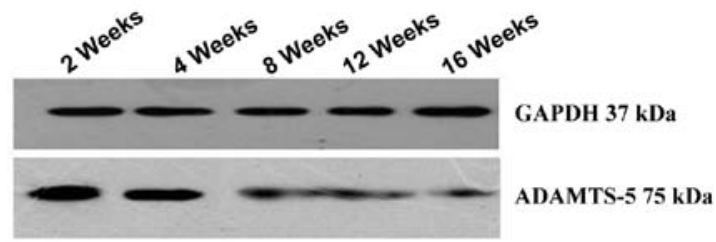

Figure 5. Transfection was successful; the protein expression level of ADAMTS-5 was downregulated, as shown by western blot analysis for ADAMTS-5 protein expression. As time progressed, ADAMTS-5 protein expression was significantly downregulated in the experimental group $(\mathrm{p}<0.05)$ compared with the normal group and the negative group.

Successful construction of lentiviral vector expressing siRNA targeting ADAMTS-5. We confirmed the succesful insertion of lentiviral vector expressing siRNA targeting ADAMTS-5 by DNA sequencing (Fig. 4A) and infected the chondrocytes at a MOI of 40 (Fig. 4B).

Western blot analysis in vivo. The protein expression of ADAMTS-5 in vivo was evaluated by western blot analysis (Fig. 5).

Histological changes in rat articular joints. All animals remained healthy during this study and no significant differences in body weight were observed among the groups (data not shown). We confirmed that ACL-T and PM surgery in all the rats induced OA-like changes in the articular cartilage. Fig. 1C-F shows the macroscopic findings in the negative control group and the blank control group at 2 and 4 weeks following surgery. In the first group (Fig. 1C and D), healthy articular cartilage was present, while in the negative control group (Fig. 1E and F) changes representing early OA were observed.
Normal cartilage has a smooth, uninterrupted surface and a uniform distribution of chondrocytes, often arranged in columns (20). A healthy appearance was observed in all the animals in the blank groups throughout the experimental period (Figs. 1 and 6). The OARSI scores demonstrated these observations and indicated that changes during early OA did not appear at any point up to 16 weeks in either joint surface (tibial or femoral) (Fig. 7).

Only slight damage to the articular cartilage (such as vertical fissures and delamination) was observed in the experimental joint surfaces at 8 and 16 weeks (Fig. 6). However, these changes did not worsen over time. The OARSI histopathological scoring demonstrated no significant progression of OA in either joint surface (Fig. 7). Our results demonstrated that the experimental group developed minor but non-advancing OA characteristics at up to 16 weeks. A direct injection of lentivirus-mediated ADAMTS-5 siRNA in the rats with OA was effective in suppressing the degeneration of articular cartilage.

Degradation in the negative control group was severe. Surface discontinuity and delamination were observed at 4 weeks (Fig. 6). A greater progression of degradation was observed in the negative control group compared to the experimental control group after 4 weeks (Fig. 6). For instance, there was a sharp increase in the breadth and depth of vertical fissures and cartilage denudation (Fig. 6).

\section{Discussion}

Despite the prevalence of OA in the elderly population, there are no effective treatments. The first-line therapy indicated for OA treatment is the analgesic, acetaminophen (21). However, it is used for pain management and has no disease-modifying benefits. The treatment of the vast majority of OA patients, particularly at the early stage of OA pathogenesis, focuses on relieving symptoms, such as pain and swelling. ADAMTS-5, a 


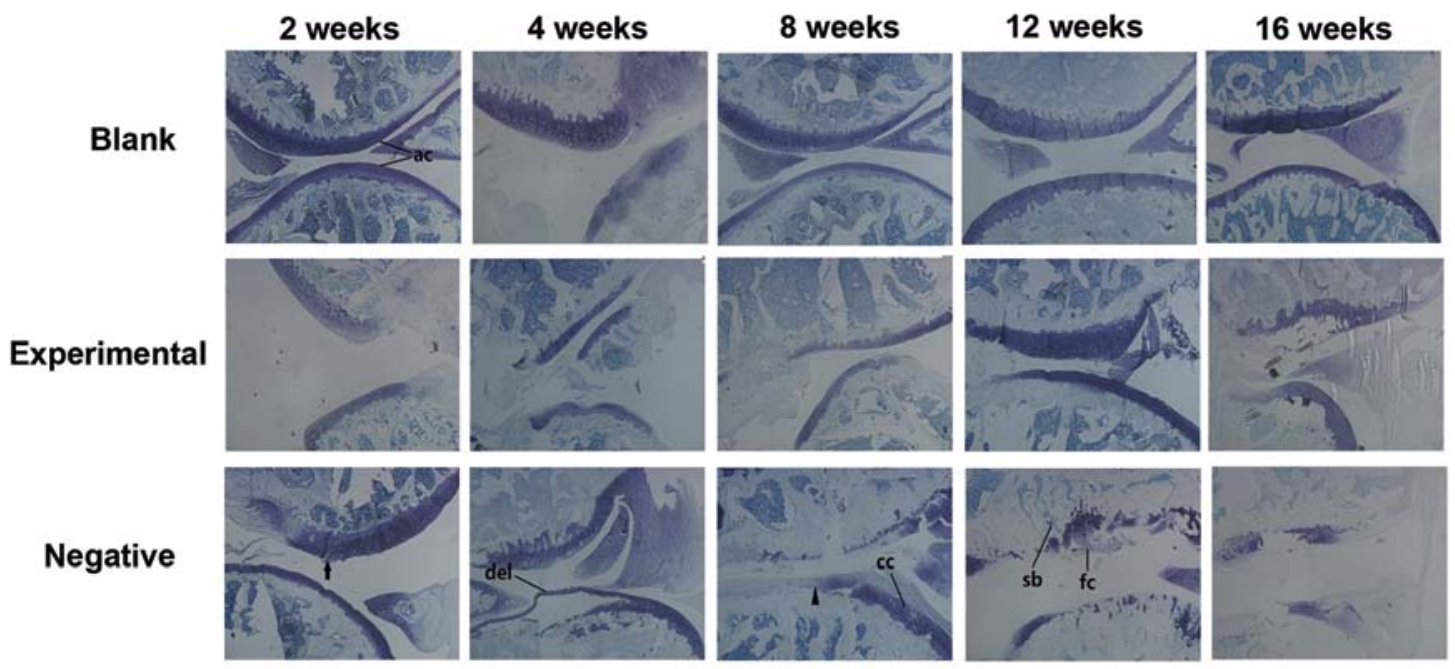

Figure 6. Histological analysis revealed patterns of articular degradation over time. Sagittal sections from the blank group, the experimental group, and the negative group of animals were analyzed over a 16-week time period. Sections were stained with toluidine blue. Knee joint tissue from each group is shown at $2,4,8,12$ and 16 weeks following surgery in the upper row of each panel. Examples of morphologically normal articular cartilage (ac), vertical fissures (black arrow head), surface discontinuity (black arrow), delamination (del), chondrocyte clusters (cc), sclerotic bone (sb) and fibrocartilage-like tissue (fc) are indicated.
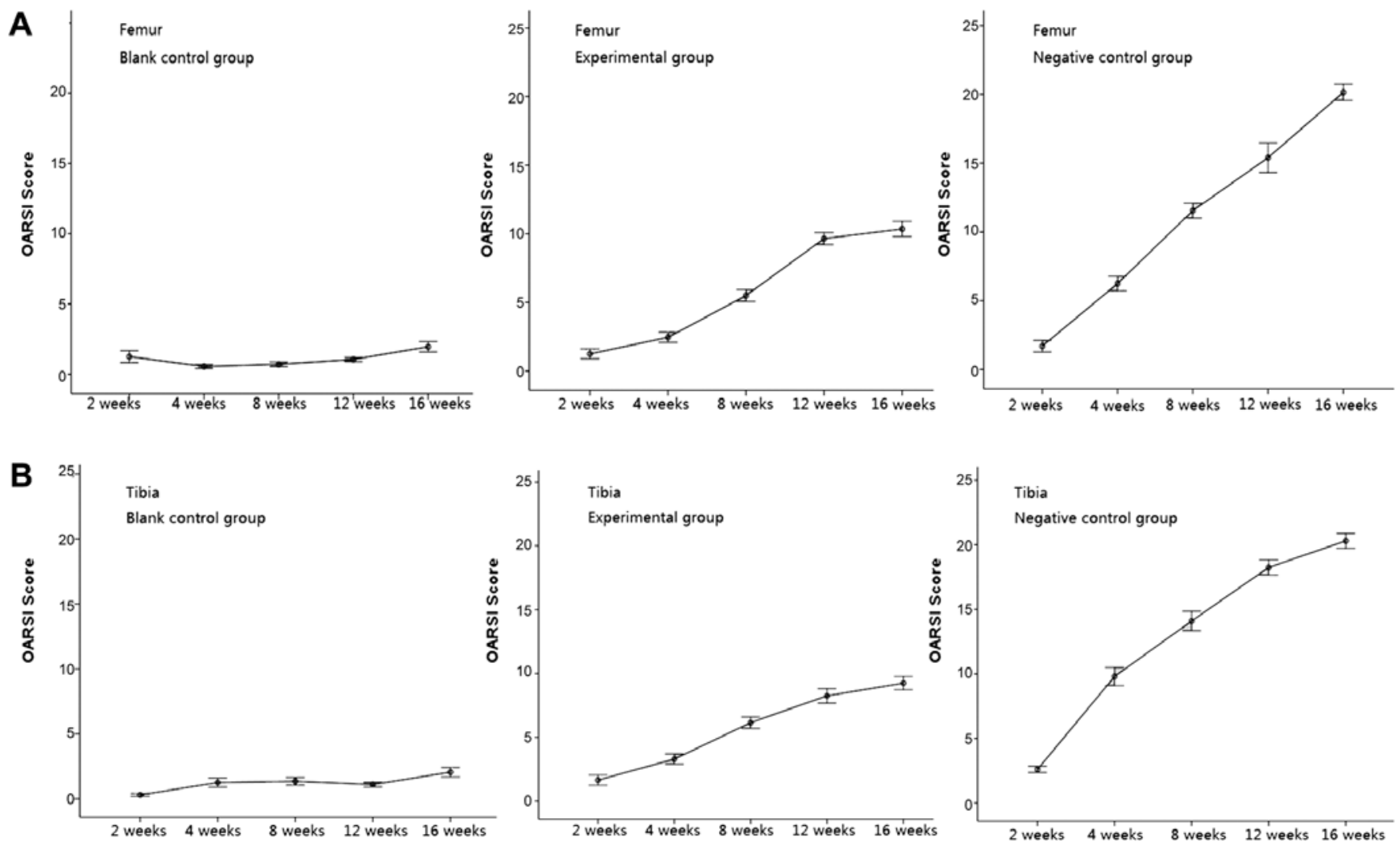

Figure 7. Osteoarthritis Research Society International (OARSI) histopathological grading and staging scores. OARSI scores were determined in the blank control group, the experimental group and the negative control group of animals over a period of 16 weeks. (A) Femur joint surfaces and (B) tibia joint surfaces in the 3 groups were assessed independently. The mean OARSI scores \pm standard error are shown. Significantly lower scores were observed in the femurs of the experimental group compared to the negative group at 8 and 16 weeks $(p<0.05)$. OARSI scores for tibial tissue of the experimental group were lower than those in the negative group at 4 weeks post-surgery.

member of the ADAMTS family, has received much attention in OA research (22). As previously reported, a single injection of ADAMTS-5 siRNA suppressed the disc degeneration of nucleus pulposus tissue in a rabbit model of anular puncture disc degeneration (13). The double-knockout of ADAMTS-4 and ADAMTS-5 in mice has been shown to prevent the progression of OA (23). These data suggest that ADAMTS-5 is the most likely candidate for a role in the pathological mechanisms of OA. 
In the present study, we hypothesized that the silencing of ADAMTS-5 expression in articular cartilage by lentivirus-mediated siRNA would inhibit the progression of OA in rats. We successfully constructed a siRNA oligonucletide for the rat ADAMTS-5 gene. According to the results of qRT-PCR and western blot analysis, ADAMTS-5 siRNA-transfected rat chondrocytes showed $>75 \%$ silencing efficiency of ADAMTS-5 mRNA compared with the control group. Furthermore, we successfully constructed lentivirus-vector-mediated siRNA targeting ADAMTS-5. Using a rat model of OA, this study investigated the effects of a direct intra-articular injection of lentivirus-mediated ADAMTS-5 siRNA into the knee on the delay and attenuation of articular cartilage degeneration.

Histological findings from toluidine blue staining demonstrated the maintenance of articular cartilage in the group treated with lentivirus-mediated ADAMTS-5 siRNA. However, the OARSI scores in the experimental group were still higher than those of the blank control group. It has been suggested that ADAMTS-5 and ADAMTS-4 are important mediators of aggrecan loss in normal cartilage (22). The silencing of ADAMTS-4 or ADAMTS-5, or both, may be worthy of further investigation. This method may lead to the development of novel therapeutic strategies for the treatment of OA in humans.

\section{Acknowledgements}

This study was supported by a grant from the Natural Science Foundation of Hubei Province (no. ZRY0558).

\section{References}

1. Sarzi-Puttini P, Cimmino MA, Scarpa R, et al: Osteoarthritis: an overview of the disease and its treatment strategies. Semin Arthritis Rheum 35 (1 Suppl 1): 1-10, 2005.

2. Hayami T, Pickarski M, Wesolowski GA, et al: The role of subchondral bone remodeling in osteoarthritis: reduction of cartilage degeneration and prevention of osteophyte formation by alendronate in the rat anterior cruciate ligament transection model. Arthritis Rheum 50: 1193-1206, 2004.

3. Stanton H, Rogerson FM, East CJ, et al: ADAMTS5 is the major aggrecanase in mouse cartilage in vivo and in vitro. Nature 434: 648-652, 2005.

4. Glasson SS, Askew R, Sheppard B, et al: Deletion of active ADAMTS5 prevents cartilage degradation in a murine model of osteoarthritis. Nature 434: 644-648, 2005.

5. Fire A, Xu S, Montgomery MK, Kostas SA, Driver SE and Mello CC: Potent and specific genetic interference by doublestranded RNA in Caenorhabditis elegans. Nature 391: 806-811, 1998.

6. Martin SE and Caplen NJ: Applications of RNA interference in mammalian systems. Annu Rev Genomics Hum Genet 8: 81-108, 2007.
7. Campbell TN and Choy FY: RNA interference: past, present and future. Curr Issues Mol Biol 7: 1-6, 2005.

8. Sen GL and Blau HM: A brief history of RNAi: the silence of the genes. FASEB J 20: 1293-1299, 2006.

9. Naldini L, Blomer U, Gallay P, et al: In vivo gene delivery and stable transduction of nondividing cells by a lentiviral vector. Science 272: 263-267, 1996.

10. Zufferey R, Dull T, Mandel RJ, et al: Self-inactivating lentivirus vector for safe and efficient in vivo gene delivery. J Virol 72: 9873-9880, 1998.

11. $\mathrm{Hu} \mathrm{DN}$, Yang $\mathrm{PY}, \mathrm{Ku} \mathrm{MC}$, Chu $\mathrm{CH}$, Lim AY and Hwang $\mathrm{MH}$ : Isolation and cultivation of human articular chondrocytes. Kaohsiung J Med Sci 18: 113-120, 2002.

12. Durigova M, Troeberg L, Nagase H, Roughley PJ and Mort JS: Involvement of ADAMTS5 and hyaluronidase in aggrecan degradation and release from OSM-stimulated cartilage. Eur Cell Mater 21: 31-45, 2011.

13. Seki S, Asanuma-Abe Y, Masuda K, et al: Effect of small interference RNA (siRNA) for ADAMTS5 on intervertebral disc degeneration in the rabbit anular needle-puncture model. Arthritis Res Ther 11: R166, 2009.

14. Hummon AB, Lim SR, Difilippantonio MJ and Ried T: Isolation and solubilization of proteins after TRIzol extraction of RNA and DNA from patient material following prolonged storage. Biotechniques 42: 467-470, 472, 2007.

15. Gouze E, Pawliuk R, Pilapil C, et al: In vivo gene delivery to synovium by lentiviral vectors. Mol Ther 5: 397-404, 2002.

16. Appleton CT, McErlain DD, Pitelka V, et al: Forced mobilization accelerates pathogenesis: characterization of a preclinical surgical model of osteoarthritis. Arthritis Res Ther 9: R13, 2007.

17. Rozas G, Guerra MJ and Labandeira-Garcia JL: An automated rotarod method for quantitative drug-free evaluation of overall motor deficits in rat models of parkinsonism. Brain Res Brain Res Protoc 2: 75-84, 1997.

18. Martins MA, de Castro Bastos L and Tonussi CR: Formalin injection into knee joints of rats: pharmacologic characterization of a deep somatic nociceptive model. J Pain 7: 100-107, 2006.

19. Custers RJ, Creemers LB, Verbout AJ, van Rijen MH, Dhert WJ and Saris DB: Reliability, reproducibility and variability of the traditional Histologic/Histochemical Grading System vs the new OARSI Osteoarthritis Cartilage Histopathology Assessment System. Osteoarthritis Cartilage 15: 1241-1248, 2007.

20. Broom ND: Further insights into the structural principles governing the function of articular cartilage. J Anat 139: 275-294, 1984.

21. Jones MD, Tran CW, Li G, Maksymowych WP, Zernicke RF and Doschak MR: In vivo microfocal computed tomography and micro-magnetic resonance imaging evaluation of antiresorptive and antiinflammatory drugs as preventive treatments of osteoarthritis in the rat. Arthritis Rheum 62: 2726-2735, 2010.

22. Song RH, Tortorella MD, Malfait AM, et al: Aggrecan degradation in human articular cartilage explants is mediated by both ADAMTS-4 and ADAMTS-5. Arthritis Rheum 56: 575-585, 2007.

23. Majumdar MK, Askew R, Schelling S, et al: Double-knockout of ADAMTS-4 and ADAMTS-5 in mice results in physiologically normal animals and prevents the progression of osteoarthritis. Arthritis Rheum 56: 3670-3674, 2007. 